\title{
Circularly polarized emission from ensembles of InAs/GaAs quantum dots
}

\author{
Evgenii Moskalenko, L.A. Larsson and Per-Olof Holtz
}

\section{Linköping University Post Print}

N.B.: When citing this work, cite the original article.

Original Publication:

Evgenii Moskalenko, L.A. Larsson and Per-Olof Holtz, Circularly polarized emission from ensembles of InAs/GaAs quantum dots, 2011, Journal of Applied Physics, (110), 1, 013510. http://dx.doi.org/10.1063/1.3599853

Copyright: American Institute of Physics http://www.aip.org/

Postprint available at: Linköping University Electronic Press http://urn.kb.se/resolve?urn=urn:nbn:se:liu:diva-69808 


\title{
Circularly polarized emission from ensembles of InAs/GaAs quantum dots
}

\author{
E. S. Moskalenko, ${ }^{1,2}$ L. A. Larsson, ${ }^{1, a)}$ and P. O. Holtz ${ }^{1}$ \\ ${ }_{1}^{1} I F M$, Material Physics, Linköping University, S-581 83 Linköping, Sweden \\ ${ }^{2}$ A. F. Ioffe Physical-Technical Institute, Russian Academy of Sciences, 194021, Polytechnicheskaya \\ 26, St. Petersburg, Russia
}

(Received 4 February 2011; accepted 13 May 2011; published online 6 July 2011)

\begin{abstract}
We present a low-temperature micro-photoluminescence ( $\mu$-PL) study of ensembles of InAs/GaAs quantum dots (QDs) with respect to its circular polarization $\left(\rho_{\mathrm{c}}\right)$ for a manifold of experimental conditions such as single or dual laser excitation, different excitation energies $\left(h v_{e x}\right)$, varying excitation powers $\left(P_{e x}\right)$ of both lasers, and with or without an external magnetic field $\left(\boldsymbol{B}_{\text {ext }}\right)$. It is demonstrated that an essential $\rho_{\mathrm{c}}(\leq 40 \%)$ could be recorded depending on $P_{\text {ex }}$, even at $\boldsymbol{B}_{\text {ext }}=0$ for $h v_{e x}$ exceeding the PL energy of the wetting layer $\left(E_{W L}\right)$, while $\rho_{\mathrm{c}}$ remains negligible for $h v_{e x}<E_{W L}$. To explain the data obtained, a model is developed according to which a nuclear magnetic field $\left(\boldsymbol{B}_{N}\right)$ is created in the QDs by spin-polarized electrons. The $\boldsymbol{B}_{\boldsymbol{N}}$ plays a crucial role in the preservation of the electron spin, which otherwise effectively relaxes due to the presence of the anisotropic electron-hole exchange interaction $\left(\omega_{\mathbf{e x}}\right)$. The application of an additional infra-red laser gives rise to a population of excess holes in the QDs, thus producing positively charged excitons. In this case, $\omega_{\mathbf{e x}}=0$ and accordingly, $\rho_{\mathrm{c}} \approx 40 \%$ at $\boldsymbol{B}_{\text {ext }}=0$ is recorded, even for excitation with $h v_{e x}<E_{W L}$. C 2011 American Institute of Physics. [doi:10.1063/1.3599853]
\end{abstract}

\section{INTRODUCTION}

The spin of an electron confined inside a semiconductor quantum dot (QD) has attracted considerable attention among researchers during the past decade, since it has been suggested to be used as a building block for future memory and quantum computer operation. ${ }^{1}$ The advantage of employing the electron spin stems from the cancellation of the classical spin relaxation mechanisms when one considers QDs. ${ }^{2}$ Experimental access to the electron spin state is easily achieved by monitoring the degree of circular polarization $\left(\rho_{\mathrm{c}}\right)$ of the emission in photoluminescence (PL) experiments. For the case of neutral excitons in QDs, a negligible $\rho_{\mathrm{c}}$ (on the order of a few \%) is predicted at zero external magnetic field $\left(\boldsymbol{B}_{\text {ext }}\right)$ due to the strong electron-hole anisotropic exchange interaction $\left(\omega_{\boldsymbol{e x}}\right){ }^{3-7}$ Conversely, for the case of charged exciton complexes, for which $\omega_{e x}$ is suppressed, an essential value for $\rho_{\mathrm{c}}$ is expected. ${ }^{5,8}$ Indeed, high values of $\rho_{\mathrm{c}}$ (from 50-95\%) were measured for charged exciton complexes in individual $\operatorname{In}(\mathrm{Ga}) \mathrm{As} / \mathrm{GaAs}$ QDs at $\boldsymbol{B}_{\text {ext }}=0,{ }^{3,9,10}$ Low values of $\rho_{\mathrm{c}}$ were recorded at $\boldsymbol{B}_{\text {ext }}=0$ for the neutral exciton PL in experiments on QD ensembles ${ }^{6,11}$ and on individual QDs. ${ }^{3,8,9}$ It is also well-known that, in the case of the neutral exciton, an external magnetic field "restores" $\rho_{\mathrm{c}}$ by suppressing $\omega_{e x}{ }^{6,7}$ However, a relatively high $\rho_{\text {c }}$ was also recently observed for the neutral exciton in individual InAs QDs at $\boldsymbol{B}_{\text {ext }}=0 .{ }^{12,13}$ This effect was explained in terms of the generation of a nuclear field $\left(\boldsymbol{B}_{N}\right)$ which effectively suppresses $\omega_{\text {ex }}$, thus playing the role of $\boldsymbol{B}_{\text {ext }}$ to "stabilize" the electron spin. It has been demonstrated ${ }^{14}$ that an efficient nuclear spin pumping could also be achieved for the neutral exciton exposed to an external magnetic field.

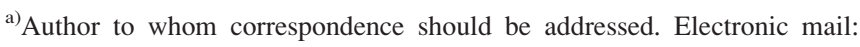
alarsson@ifm.liu.se.
}

In this paper, we present a comprehensive study of the circularly polarized PL from ensembles of a single layer of InAs/GaAs QDs grown on a wetting layer (WL) in a nominally undoped sample: A wide range of excitation powers $\left(P_{e x}\right)$, laser excitation energies $\left(h v_{e x}\right)$, and magnetic fields were employed. Experiments with a single laser excitation were complemented with dual laser excitation conditions, when a second infrared (IR) laser is used in addition to the main laser. It is demonstrated that at single laser excitation conditions and $\boldsymbol{B}_{\text {ext }}=0$, the PL emitted from an ensemble of neutral QDs is circularly polarized. The $\rho_{\mathrm{c}}$ is found to increase from 4 to $\approx 40 \%$ when increasing $P_{e x}$, under the experimental conditions when $h v_{e x}$ is tuned above the WL peak energy $\left(E_{W L}\right)$. On the contrary, at $h v_{e x}<E_{W L}, \rho_{\mathrm{c}}$ remains small (2-10\%) in the entire $P_{e x}$ range used. An external magnetic field applied in the Faraday geometry can essentially increase $\rho_{\mathrm{c}}$ (up to $\approx 10$ times).

The results obtained are satisfactorily explained by the model developed, according to which, electrons and holes are captured separately from the WL into the QDs. This results in a time delay $\left(\Delta \tau_{\mathrm{e}-\mathrm{h}}\right)$, during which an electron is trapped inside the QD alone (before the hole is captured), and can polarize the nuclei with a certain probability. As a result, a nuclear field is established, which in turn "stabilizes" the electron spin, preserving it from the "destructive" influence of $\boldsymbol{\omega}_{\boldsymbol{e x}}$. The $\boldsymbol{B}_{\boldsymbol{N}}$ is believed to be entirely responsible for the high $\rho_{\mathrm{c}}(\leq 40 \%)$ recorded for neutral excitons at $\boldsymbol{B}_{\text {ext }}=0$. Evidently, at the excitation directly into the QDs, i.e., with $h v_{e x}<E_{W L}, \Delta \tau_{\text {e-h }}$ is assumed to be zero and hence, no $\boldsymbol{B}_{\boldsymbol{N}}$ could develop, which in turn should result in negligible values of $\rho_{\mathrm{c}}$.

The application of an additional IR laser together with the main laser, exciting QDs at $h v_{e x}<E_{W L}$, increases $\rho_{\mathrm{c}}$ considerably (from 7 to $43 \%$ ) even at $\boldsymbol{B}_{\text {ext }}=0$. This fact is explained by the creation of positively charged excitons, for 
which $\omega_{e x}=0$ in the QDs, because illumination of the sample with solely IR excitation creates free holes due to excitation into deep levels in the GaAs barriers. ${ }^{15}$

\section{Samples and experimental setup}

The sample under study was grown by molecular beam epitaxy and consists of an originally 1.7 monolayer thick InAs WL and the InAs QDs positioned between two GaAs barriers (a detailed sample description is given in Refs. 16 and 17). A diffraction-limited micro-photoluminescence ( $\mu$-PL) setup was employed to study different parts of the sample with different QD densities. A Ti:sapphire laser beam was focused down to a spot diameter of $2 \mu \mathrm{m}$ on the sample surface by means of a micro-objective. The excitation energy $\left(h \nu_{\mathrm{ex}}\right)$ of the laser could be tuned in the range from 1.23 to $1.77 \mathrm{eV}$ with a maximum excitation power $\left(P_{e x}\right)$ of $3 \mathrm{~mW}$. A second semiconductor IR laser, operating at an excitation energy $h \nu_{\mathrm{IR}}=1.17 \mathrm{eV}$ with a maximum power $\left(P_{I R}\right)$ of $\approx 1 \mathrm{~mW}$, was used to perform dual laser excitation experiments. The sample was positioned inside a continuous-flow cryostat, which allowed the temperature $(T)$ to be altered from 3.8 up to $300 \mathrm{~K}$. All presented data was measured at $T=4 \mathrm{~K}$. The cryostat was inserted into the center of a superconducting solenoid providing the possibility of applying a magnetic field up to $5 \mathrm{~T}$.

To excite the sample with circularly polarized light $\left(\sigma^{+}\right.$and/or $\left.\sigma^{-}\right)$, the linearly polarized laser beam was passed through a quarter-wave plate (Berek compensator) providing a high degree $(\approx 98-99 \%)$ of circular polarization of the exciting light. The PL signal was collected through the same micro-objective and passed through another quarter wave plate combined with a Glan-Thomson linear polarizer (to analyze the PL signal with respect to its circular polarization) positioned before the entrance slits of the spectrometer. A single-grating $0.55-\mathrm{m}$ monochromator, combined with a nitrogen-cooled CCD camera, allowed a spectral resolution of $\approx 75 \mu \mathrm{eV}$. The degree of circular polarization $\left(\rho_{\mathrm{c}}\right)$ of the PL was determined from $\rho_{\mathrm{c}}=\left(I_{\text {co }}-I_{\text {cross }}\right) /\left(I_{\text {co }}+I_{\text {cross }}\right)$, where $I_{c o}\left(I_{\text {cross }}\right)$ stands for the spectrally integrated co(cross-) circularly polarized PL component.

Seven different parts of the sample with different QD densities were studied, and all of them show similar results. Here we present experimental data for one specific spot of the sample for consistency.

\section{EXPERIMENTAL RESULTS AND DISCUSSION}

Figure 1(a) shows a $\mu$-PL spectrum of the sample consisting of the WL emission band peaking at $E_{W L}=1.45 \mathrm{eV}$ and the QD emission, which appears as a broadband centered at $1.26 \mathrm{eV}$. The $\mu$-PL excitation (PLE) spectrum of the WL shows, in addition to the GaAs exciton absorption peak, two peaks labeled $\mathrm{HH}$ and $\mathrm{LH}$ (with spectral positions denoted $E_{H H}$ and $E_{L H}$ ), which were previously identified ${ }^{18}$ as transitions from the heavy- and light-hole levels in the WL.

A large number of sharp emission lines $(\approx 100)$ can be distinguished within the spectral region of the QD emission [Fig. 1(a)], indicating that the present ensemble of QDs has an areal density corresponding to approximately $N \approx 100$
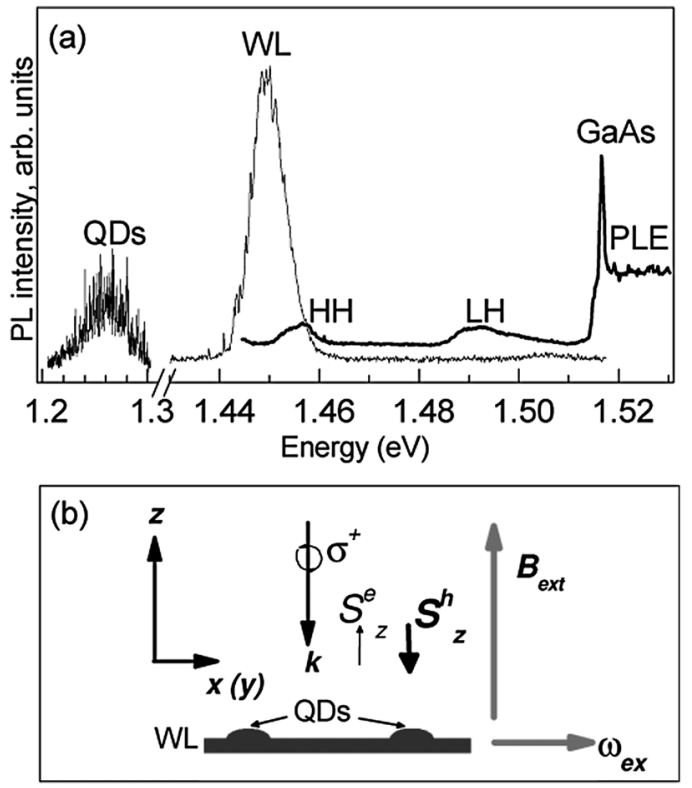

FIG. 1. (a) $\mu$-PL spectrum of the sample (thin line) measured at $h v_{e x}=1.745$ $\mathrm{eV}$ and $P_{e x}=20 \mathrm{nW}$. The thick line shows the PLE spectrum of the WL detected at the low-energy part of the WL PL-band at $P_{e x}=2 \mathrm{~mW}$. All data are obtained at $B_{e x t}=0 \mathrm{~T}$. (b) Schematic illustration of the experiment.

QDs within the area of the laser spot. The value of $N$ could also be estimated from a different procedure, namely by comparing the spectrally integrated PL intensities of the QDs $\left(I_{Q D}\right)$ and the WL $\left(I_{W L}\right)$. Indeed, carriers photo-excited in the GaAs barriers with a generation rate, $G$, can be captured into the QDs (with a capture rate, $\gamma_{c}$ ) or relax to the lowest WL energy state (with relaxation rate, $\gamma_{\mathrm{r}}$ ) prior to recombination. Based on simple rate equations (similar to those in Ref. 15), it follows that $I_{Q D}=G \gamma_{\mathrm{c}} /\left(\gamma_{\mathrm{c}}+\gamma_{\mathrm{r}}\right)$ and $I_{W L}=G \gamma_{\mathrm{r}} /\left(\gamma_{\mathrm{c}}+\gamma_{\mathrm{r}}\right)$ and hence, $I_{Q D} / I_{W L}=R=\gamma_{\mathrm{c}} / \gamma_{\mathrm{r}}$. In our previous studies ${ }^{19}$ of single QDs on the same sample (i.e., only one QD located within the laser spot), $R$ as large as $R \approx 0.01$ was derived and allowed us to estimate the value of $\gamma_{\mathrm{c}}$ for a single QD to be $\gamma_{c}^{S} \approx 0.01 \gamma_{\mathrm{r}}$. Assuming (i) that $\gamma_{\mathrm{r}}$ is independent of $N$ (which is reasonable since the probability for carrier energy relaxation is entirely determined by coupling with phonons), and (ii) that $\gamma_{c}$ is directly proportional to the QD density, i.e., $\gamma_{\mathrm{c}}=\mathrm{N} \gamma_{c}^{S}$, one could easily estimate $N$ when $R$ is given. Based on the data shown in Fig. 1 (a), $R \approx 1.4$ is deduced, resulting in $N \approx 140$, which is rather close to the value $N \approx 100$ peviously derived.

The estimates that $N \approx 100-140$, corresponding to an areal density of QDs $\approx 2.5-3.5 \times 10^{9} \mathrm{~cm}^{-2}$, allow us to consider the charge state of the ensemble of QDs under study to be neutral at a single laser excitation. Indeed, in our previous study on the charging of single QDs with extra electrons by pure optical means performed on another part of the same sample, the concentration of residual impurities in our nonintentionally-doped sample was estimated to be $\approx 10^{13}$ $\mathrm{cm}^{-3}{ }^{18}$ This shows that the total number of extra (noncompensated) carriers which could be created by the laser within the excited volume $\left[\approx 6.3 \times 10^{-13} \mathrm{~cm}^{3}\right.$ (Ref. 20)] of the GaAs barrier, is $\approx 2-3$ in steady-state conditions. This is evidently insufficient to charge the QD ensemble under study, i.e., with 100-140 QDs within the laser spot. Conversely, to 
study negatively ${ }^{11,21}$ or positively ${ }^{5}$ charged ensembles of QDs, intentional doping of the sample with a dopant density similar to the QD density is required.

Contrary to the data shown in Fig. 1(a), with linearly polarized excitation, the experimental results obtained employing circularly polarized $\left(\sigma^{+}\right)$excitation are presented in Fig. 2. Similar data were obtained for $\sigma^{-}$excitation (not shown here). The $\sigma^{+}$polarized excitation, with wave vector, $\boldsymbol{k}$, propagates along the growth direction $(\boldsymbol{z})$. Upon absorption, the excitation creates electrons (holes) with spin projection antiparallel (parallel) to $\boldsymbol{k}$ as schematically shown in Fig. 1(b). If the spin projections of the photo-excited electrons and holes are conserved, at least partially, during the capture into and the relaxation inside the QDs, the emitted photons will carry a nonzero $\rho_{\mathrm{c}}$, which, in fact, directly measures the spin projection of the recombining particles along the $z$ axis. It should be noted that the laser excitation energy, $E_{H H}<h v_{e x}<E_{L H}$, was chosen to obtain the spectra in Fig. 2 in order to solely excite carriers with a definite sign of their spin projection onto the $z$ axis. Indeed, when the laser excites only the $\mathrm{HH}$ state, say, with $\sigma^{+}$polarization, it creates electrons (holes) with spin projection onto the $z$ axis, $S_{z}^{e},\left(S_{z}^{h}\right)$ of $+1 / 2(-3 / 2)$, while in the case of $h v_{e x} \geq E_{L H}$, electrons and holes with $S_{z}^{e}=-1 / 2$ and $S_{z}^{h}=-1 / 2$ are also photo-

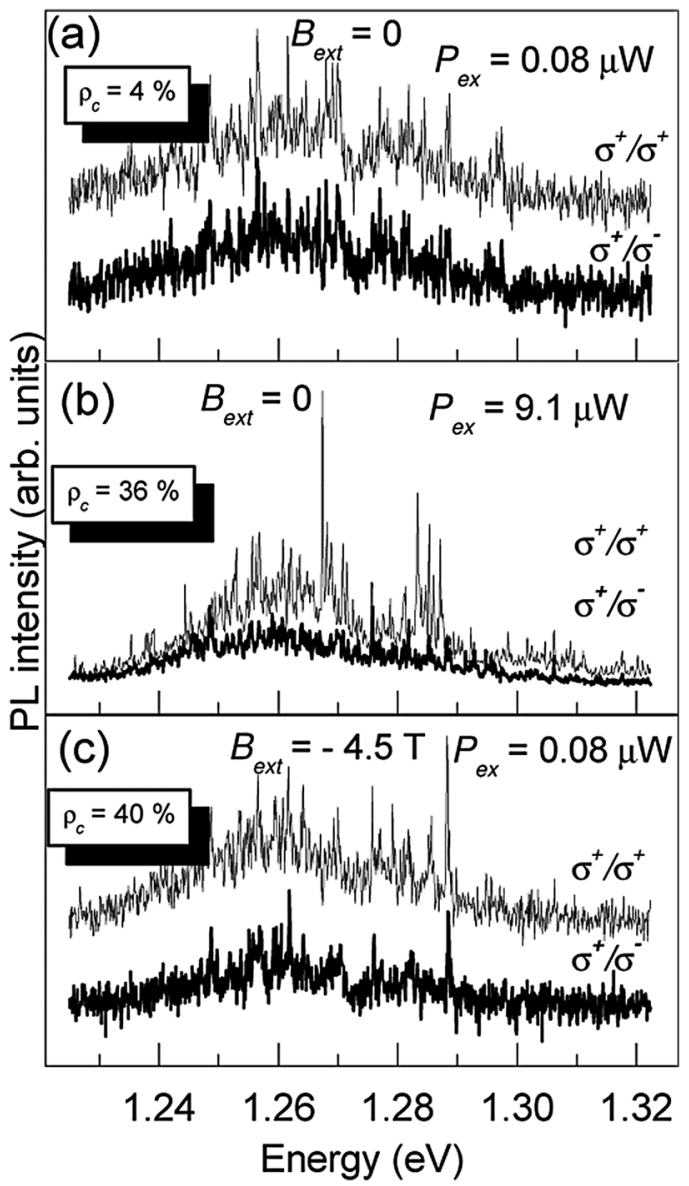

FIG. 2. $\mu$-PL spectra of the QDs measured at $h v_{e x}=1.465 \mathrm{eV}$, (a) $B_{\text {ext }}=0 \mathrm{~T}$ and $P_{e x}=0.08 \mu \mathrm{W}$, (b) $B_{e x t}=0 \mathrm{~T}$ and $P_{e x}=9.1 \mu \mathrm{W}$, (c) $B_{e x t}=-4.5 \mathrm{~T}$ and $P_{e x}=0.08 \mu \mathrm{W}$. The labeling of the thin (thick) lines, $\sigma^{+} / \sigma^{+}\left(\sigma^{+} / \sigma^{-}\right)$, denotes $\sigma^{+}$-polarized excitation and co- (cross-) polarized detection. In (a) and (c) the spectra have been vertically shifted for clarity. excited. $^{22}$ It is a widely accepted assumption that, upon excitation into the WL, the electron spin does not relax during capture into the QD, whereas the initial hole spin orientation is lost. ${ }^{5,11,23}$ Hence, the remaining spin projection of the recombining exciton is entirely determined by the preserved electron spin projection. It follows that at excitation with $h v_{e x} \geq E_{L H}$, no exciton polarization is predicted, which is, indeed, confirmed in our experiments (not shown here).

Figure 2(a) shows two $\mu$-PL spectra recorded at $\boldsymbol{B}_{\text {ext }}=0$ with the polarization selection in the detection path (as indicated in the figure) at the lowest laser excitation power, $P_{e x}=0.08 \mu \mathrm{W}$. These spectra are almost identical with a rather limited polarization, $\rho_{\mathrm{c}} \approx 0.04$. A new spectrum measured at the same experimental conditions, however, with an increased $P_{e x}=9.1 \mu \mathrm{W}$ give an increase of $\rho_{\mathrm{c}}$ by approximately 10 times [Fig. 2(b)]. The evolution of $\rho_{\mathrm{c}}$ with increasing $P_{e x}$ reveals a gradual increase up to $P_{e x} \approx 10 \mu \mathrm{W}$, followed by a slight decrease [solid symbols in Fig. 3(a)]. Based on these results, one can conclude that the spin of the electrons is preserved more effectively at high $P_{e x}$.

To further study the electron spin preservation phenomena, the QDs were excited with $h v_{e x}=1.424 \mathrm{eV}$ which is essentially less than any transitions related to the WL [see Fig. 1(a)] and hence corresponds to excitation directly into the QDs. These experimental conditions are expected to facilitate electron spin preservation since no carrier transport is needed prior to their capture into the QDs. Consequently, a similar or even higher $\rho_{\mathrm{c}}$ compared to the case of excitation with $h v_{e x} \geq E_{H H}$ is predicted. Evidently, the experimental data [Fig. 3(a)] contradicts this expectation: $\rho_{\mathrm{c}}$ does not exceed 0.1 for any $P_{e x}$. It is important to stress here that a
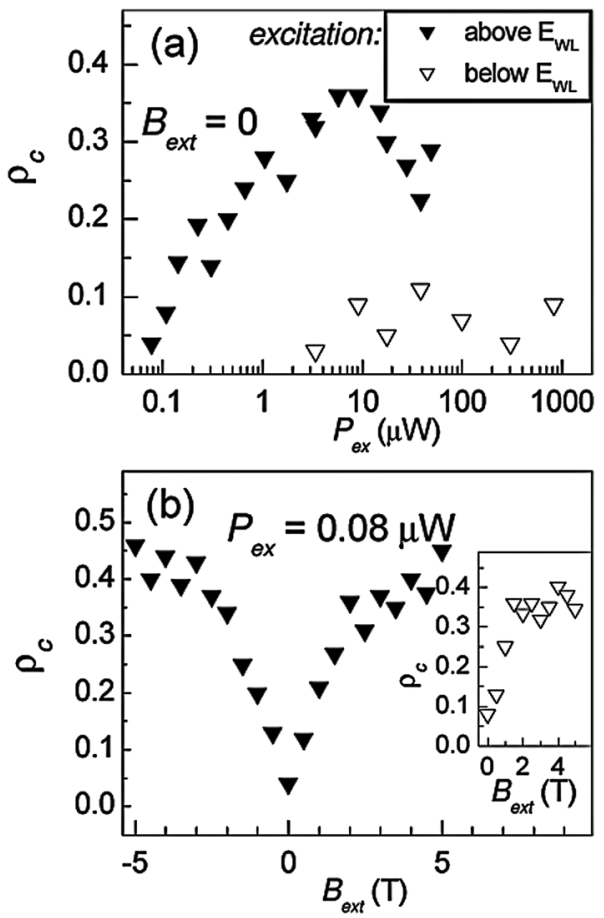

FIG. 3. (a) The solid (open) symbols show $\rho_{\mathrm{c}}$ as a function of $P_{e x}$ recorded at $\sigma^{+} \mathrm{cw}$ excitation for $h v_{e x}=1.465(1.424) \mathrm{eV}$ and $B_{e x t}=0$. (b) $\rho_{\mathrm{c}}$ as a function of $B_{e x t}$ recorded at $\sigma^{+} \mathrm{cw}$ excitation for $h v_{e x}=1.465 \mathrm{eV}$ and $P_{e x}=0.08$ $\mu \mathrm{W}$. The inset in (b) shows $\rho_{\mathrm{c}}$ as a function of $B_{\text {ext }}$ recorded at $\sigma^{+}$cw excitation for $h v_{e x}=1.424 \mathrm{eV}$ and $P_{e x}=50 \mu \mathrm{W}$. 
decrease in absorption at these experimental conditions compared to the case of excitation with $h v_{e x} \geq E_{H H}$ can be ruled out as the possible reason for the observed small $\rho_{\mathrm{c}}$ [Fig. 3(a)]. This is based on the fact that $I_{Q D}$ at $h v_{e x}=1.424$ $\mathrm{eV}\left(I_{Q D}{ }^{d i r}\right)$ is only $\approx 5$ times smaller than that recorded at $h v_{e x} \geq E_{H H}\left(I_{Q D}{ }^{\text {above }}\right)$ for the same $P_{e x}{ }^{24}$

Accordingly, it can be concluded that an elaborate mechanism of the electron spin relaxation is required, which takes into account the preferential spin preservation, both for the excitation at $h v_{e x} \geq E_{H H}$ (as compared with excitation direct into the QDs) and at the high $P_{e x}$ employed (as compared with low $P_{e x}$ ) to explain the experimental data [Figs. 2(a), 2(b) and 3(a)]. To obtain insight into the electron/exciton spin relaxation we rely on the fine structure of the heavyhole neutral exciton in the $\mathrm{QDs}^{25}$ composed of an electron and a hole with $S_{z}^{e}= \pm 1 / 2$ and $S_{z}^{h}= \pm 3 / 2$. When combining $S_{z}^{e}$ and $S_{z}^{h}$, four excitonic states $(m)$ can be formed: $m=|-2\rangle$, $|-1\rangle,|+1\rangle$ and $|+2\rangle$ corresponding to $\left(S_{z}^{e}, S_{z}^{h}\right)$ combinations of the form $(-1 / 2,-3 / 2),(+1 / 2,-3 / 2),(-1 / 2,+3 / 2)$ and $(+1 / 2,+3 / 2)$. The states $|-1\rangle$ and $|+1\rangle$ are called "bright" because they directly couple to light, emitting and absorbing photons with $\sigma^{+}$and $\sigma^{-}$helicities, respectively, while the other two $|-2\rangle$ and $|+2\rangle$ are called "dark" since they are optically inactive and hence, will not be considered in the following. It is well-known that the electron-hole exchange interaction, $\omega_{e x}$, which couples the electron and hole spins, mixes a pair of bright states in the case of an inplane asymmetry of a QD. ${ }^{26}$ This creates two linearly polarized dipoles $|\mathrm{X}\rangle=2^{-[1 / 2]}(|+1\rangle+|-1\rangle)$ and $|\mathrm{Y}|=2^{-[1 / 2]}$ $(|+1\rangle-|-1\rangle) / i$, which, in the case of InAs/GaAs QDs emit light along the $\langle 110\rangle$ and $\langle 110\rangle$ crystallographic directions, ${ }^{27}$ respectively. These states are split by the anisotropic electron-hole exchange energy $\left(\hbar \omega_{\mathrm{ex}}\right)$. Circularly polarized light excites a coherent superposition of the $|\mathrm{X}\rangle$ and $|\mathrm{Y}\rangle$ states with the subsequent time evolution driven by the anisotropic exchange field resulting in quantum beats between these states on a typical time scale $\left(\tau_{\mathrm{b}}\right)$ given by $\tau_{\mathrm{b}}=2 \pi / \omega_{\boldsymbol{e x}}{ }^{6}{ }^{6}$ These quantum beats are equivalent to the precession of the initial exciton spin $\left(\boldsymbol{S}_{\mathbf{0}}^{e x}\right)$ directed along the $\boldsymbol{z}$ axis around the effective magnetic field $\left(\omega_{e x}\right)$ directed perpendicularly to the $z$ axis, according to the vector model for the exciton pseudo$\operatorname{spin}^{4,7}$ [see Fig. 1(b)]. If the value of $\tau_{b}$ is essentially less than the exciton decay time $\left(\tau_{\mathrm{d}}\right), \boldsymbol{S}_{\mathbf{0}}^{\boldsymbol{e x}}$ will accomplish many turns around $\omega_{e x}$ before it recombines and hence, $\rho_{\mathrm{c}}$ (which is proportional to the value of the projection of $S_{0}^{e x}$ onto the $z$ axis) will be negligible. ${ }^{4,6,7}$ Thus, assuming $\hbar \omega_{\mathrm{ex}} \approx 10-100$ $\mu \mathrm{eV}$ (as was experimentally derived for the neutral exciton in individual InAs/GaAs and $\mathrm{InGaAs} / \mathrm{GaAs} \mathrm{QDs}^{8,10,26}$ ) one evaluates $\tau_{\mathrm{b}} \approx 40-400$ ps. Adopting $\tau_{\mathrm{d}} \approx 800$ ps (Ref. 27) will result in $\rho_{\mathrm{c}}<1 \%{ }^{28}$ It is important to note that the ground state of single negatively (positively) charged exciton complexes consists of spin-paired electrons (holes), which effectively "switches off" $\omega_{\boldsymbol{e x}}$. As a result, high values of $\rho_{\mathrm{c}}$ (from 50 to $95 \%$ ) were measured for the charged exciton complexes in individual In $(\mathrm{Ga}) \mathrm{As} / \mathrm{GaAs}$ QDs at $\boldsymbol{B}_{\text {ext }}=0.0^{3,9,10}$

It has also been well documented ${ }^{6,7}$ that an external magnetic field of sufficient strength $\left(\left|\boldsymbol{B}_{\text {ext }}\right| \mu_{\mathrm{B}} \mathrm{g}_{\mathrm{ex}}>\hbar \omega_{\mathrm{ex}}\right.$, where $\mu_{\mathrm{B}} \approx 58 \mu \mathrm{eV} / \mathrm{T}$ is the Bohr magneton, and $g_{\text {ex }}$ stands for the $g$-factor of the neutral exciton), applied in Faraday geometry $\left(\boldsymbol{B}_{\text {ext }} \| \boldsymbol{z}\right)$ "restores" the polarization of the neutral exciton, because of the decoupling of the $|+1\rangle$ and $|-1\rangle$ states. In other words, with an increased $\left|\boldsymbol{B}_{\text {ext }}\right|$, the mixed states, $|\mathrm{X}\rangle$ and $|\mathrm{Y}\rangle$, transform into the "pure" states $|+1\rangle$ and $|-1\rangle$, thus giving rise to a nonvanishing $\rho_{\mathrm{c}}$. In the framework of the exciton pseudospin model, ${ }^{4,7}$ the initial exciton pseudospin $S_{0}^{e x}$ rotates around the total effective magnetic field $\boldsymbol{B}=\boldsymbol{B}_{\text {ext }}+\omega_{\mathrm{ex}}$, which preserves the projection of $\boldsymbol{S}_{\mathbf{0}}^{\boldsymbol{e x}}$ onto $\boldsymbol{B}$. This eventually leads to a nonzero projection of $\boldsymbol{S}_{\mathbf{0}}^{\boldsymbol{e x}}$ onto the $\boldsymbol{z}$ axis, at elevated $\boldsymbol{B}_{\text {ext }}$.

Polarization-resolved $\mu$-PL spectra, measured with the same low $P_{e x}$ as used in Fig. 2(a), but in the presence of $\boldsymbol{B}_{\text {ext }}$, provide a significantly higher polarization degree, $\rho_{\mathrm{c}} \approx 40 \%$ [Fig. 2(c)]. The gradual increase of $\rho_{\mathrm{c}}$ with increasing $\boldsymbol{B}_{\text {ext }}$, recorded at low $P_{e x}$ and at $h v_{e x} \geq E_{H H}$, is independent of the direction of $\boldsymbol{B}_{\text {ext }}$ [Fig. 3(b)]. The same behavior of $\rho_{\mathrm{c}}$ is also observed in the case of excitation directly into the QD [inset in Fig. 3(b)]. This indicates that an external field, $\boldsymbol{B}_{\text {ext }}$, on the order of $1 \mathrm{~T}$ is sufficient to start the transformation of the $|\mathrm{X}|$ and $|\mathrm{Y}|$ states into the "pure" $|+1\rangle$ and $|-1\rangle$ states. Indeed, adopting $g_{\text {ex }} \approx 3$, as estimated by others for InAs/GaAs $\mathrm{QDs},{ }^{26}\left|\boldsymbol{B}_{\text {ext }}\right| \mu_{\mathrm{B}} \mathrm{g}_{\mathrm{ex}} \approx 174 \mu \mathrm{eV}$ is calculated for $\left|\boldsymbol{B}_{\text {ext }}\right|=1 \mathrm{~T}$ which exceeds $\hbar \omega_{\mathrm{ex}} \approx 10-100 \mu \mathrm{eV}$ previously introduced.

Clearly, the exciton pseudospin model nicely describes the properties recorded both at excitation directly into the QDs and at excitation above the WL at lower power, but fails to explain the results shown in Figs. 2(b) and 3(a). Comparing the results shown in Figs. 3(a) and 3(b) for $h v_{e x} \geq E_{H H}$, it is obvious that the increase of $P_{e x}$ at $\boldsymbol{B}_{\text {ext }}=0$ [Fig. 3(a)] affects $\rho_{\mathrm{c}}$ similarly to $\boldsymbol{B}_{\text {ext }}$ [Fig. 3(b)]. Hence, one can intuitively conclude that the increase of $P_{e x}$ creates an effective magnetic field $\left(\boldsymbol{B}_{\text {eff }}\right)$ in the sample. According to the data shown in Fig. 3(b), the magnitude of this field should be as large as 2-3 T to achieve $\rho_{\mathrm{c}} \approx 36 \%$ recorded at $\boldsymbol{B}_{\text {ext }}=0$ and $P_{\text {ex }} \approx 10 \mu \mathrm{W}$ [Fig. 3(a)]. It should be emphasized that $\boldsymbol{B}_{\text {eff }}$ can be created solely at excitation with $h v_{e x} \geq E_{H H}$, according to the data shown in Fig. 3(a).

To explain the origin of $\boldsymbol{B}_{\text {eff }}$, we use the concept of dynamic polarization of the lattice nuclei created by optically oriented electrons (see Ref. 22). This effect stems from the coupling of electron and nuclear spins through the contact hyperfine Fermi interaction. The interaction of the nuclear spins with a hole is, on the contrary, considerably weaker (due to the p-type hole Bloch functions ${ }^{22}$ ), and will be excluded from further discussion.

The possibility of polarizing the nuclei in a metal by an electron spin system in equilibrium was first pointed out by Overhauser, by exerting a metal to an external magnetic field and microwave irradiation (the classical Overhauser effect). ${ }^{29}$ If optically generated electrons have a nonzero time-averaged spin $(\boldsymbol{S})$, this results in a dynamic nuclear polarization. The optical dynamic nuclear polarization in semiconductors was first detected using the conventional nuclear magnetic resonance in bulk silicon. ${ }^{30}$ An optically detected nuclear magnetic field (relying on the photoluminescence polarization) was further demonstrated in bulk GaAs crystals. ${ }^{31}$ The dynamic nuclear polarization in $2 \mathrm{D}$ systems has also been widely studied, for example, in GaAs/ AlGaAs quantum wells ${ }^{32,33}$ and in GaInP epitaxial layers. ${ }^{34}$ 
The experiments on optical orientation of the electron-nuclear spin system in $2 \mathrm{D}, 1 \mathrm{D}$, and $0 \mathrm{D}$ semiconductor structures are comprehensively reviewed in Refs. 1 and 35.

In structures of higher dimensionalities (with respect to QDs), a small but nonzero $\boldsymbol{B}_{\text {ext }}$ was needed to optically induce an essential nuclear polarization. The value of $\boldsymbol{B}_{\text {ext }}$ should exceed typical magnitudes of local effective magnetic fields $\left(B_{L} \approx 0.1-0.3 \mathrm{mT}^{22}\right)$ caused by nuclear spin-spin interactions, which lead to the disappearance of optically oriented nuclear spins. Clearly, the role of $\boldsymbol{B}_{\text {ext }}$ is to "preserve" the nuclear polarization. In our case, we assume that a significant nuclear polarization could develop, even at $\boldsymbol{B}_{\text {ext }}=0$, which represents an evident contradiction to the above considerations. To clarify the situation, we emphasize that a strongly coupled electron-nuclear spin system gives rise to two effective magnetic fields: a nuclear field $\left(\boldsymbol{B}_{N}\right)$ acting upon the electrons, and an electron field $\left(\boldsymbol{B}_{e}\right)$, called the Knight field, acting upon the nuclei. ${ }^{22}$ If $\left|\boldsymbol{B}_{\boldsymbol{e}}\right|>B_{L}$, a dynamic nuclear polarization is predicted, even at $\boldsymbol{B}_{\text {ext }}=0$.

The electron field, $\boldsymbol{B}_{\boldsymbol{e}}$, is related to $\rho_{\mathrm{c}}$ in the following way: $\left|\boldsymbol{B}_{\boldsymbol{e}}^{z}\right|=b_{e} \times\left|\boldsymbol{S}_{z}\right|=[1 / 2]\left|b_{e}\right| \times \rho_{\mathrm{c}}$, where $\boldsymbol{B}_{\boldsymbol{e}}^{z}$ and $\boldsymbol{S}_{z}$ are projections of the time-averaged electron field and the electron spin $(\boldsymbol{S})$ onto the $z$ axis, respectively, and $b_{e}$ is a parameter to be evaluated in the following text. The average interaction energy of an electron spin, $S$ (with respect to the nuclear state), with $N$ nuclei of the same species, assuming their mean spins $\left(\boldsymbol{I}_{\boldsymbol{a v}}\right)$ are equal, can be expressed as $A \boldsymbol{I}_{\boldsymbol{a}} \boldsymbol{V} \boldsymbol{S},{ }^{22}$ where $A$ is the hyperfine interaction constant. Taking $A \boldsymbol{I}_{\boldsymbol{a}} \boldsymbol{S}$ as the nuclear spin energy in an effective electron field, $\boldsymbol{B}_{\boldsymbol{e}}$, one obtains: $A \boldsymbol{I}_{a v} \boldsymbol{S}=-N \hbar \gamma \mathbf{I}_{\mathrm{av}} \mathbf{B}_{\mathbf{e}}$ (Ref. 22) and hence, $\boldsymbol{B}_{\boldsymbol{e}}=-\boldsymbol{S A} /(N \hbar \gamma)$, where $\gamma$ is the nuclear gyromagnetic ratio. The magnetic moment of a nuclei of type $i\left(\mu_{\mathrm{i}}\right)$ is connected to its gyromagnetic ratio $\left(\gamma_{\mathrm{i}}\right)$ via $\mu_{\mathrm{i}}=\hbar \gamma_{\mathrm{i}} I_{\mathrm{i}}$, where $I_{i}$ is the nuclear spin of the type $i$ nucleus. For In and As, one has $I_{I n}=9 / 2$ and $I_{A s}=3 / 2$, respectively. ${ }^{3}$ Taking into account the different nuclear species in InAs, we estimate $A / \gamma$ as $[1 / 2] \Sigma_{i}\left(A_{i} / \gamma_{\mathrm{i}}\right)$. Since the total number of nuclei in the QDs under study is not known, it is assumed that $N \approx 5 \times 10^{4}$, relying on the widely accepted estimate of $N$ for a typical $\mathrm{In}(\mathrm{Ga}) \mathrm{As} / \mathrm{GaAs}$ QD (Ref. 3). Using $A_{I n}\left(A_{A s}\right)=56$ (46) $\mu \mathrm{eV}$ (Ref. 3) and $\gamma_{\text {In }}\left(\gamma_{\mathrm{As}}\right)=5.86(4.58) \times 10^{7} \mathrm{rad} \mathrm{T}^{-1} \mathrm{~s}^{-1}, 36$ one gets $b_{e} \approx-30 \mathrm{mT}$ and hence, $\left|\boldsymbol{B}_{e}^{z}\right| \approx 15 \mathrm{mT}$ for a fully polarized electron spin $\left(\rho_{\mathrm{c}}=1\right)$. This value agrees well with estimates made by others of the magnitude of the Knight field. ${ }^{10}$ For our experimental conditions $\left(\rho_{\mathrm{c}} \approx 0.40\right)$, a field, $\left|\boldsymbol{B}_{e}^{z}\right|$, on the order of $6 \mathrm{mT}$ has been predicted and developed for similar QDs studied here. This value is well above the typical magnitude of $B_{L}$ (see preceding text), a fact that justifies our assumption that an essential $\boldsymbol{B}_{N}$ could be developed in the QD under study even at $\boldsymbol{B}_{\text {ext }}=0$.

As a result, we assume that the interaction of a single electron and a large number of nuclei $\left(10^{4}-10^{5}\right)$ in a single QD results in a dynamic nuclear spin polarization. This gives rise to the appearance of spin-oriented nuclei, which is equivalent to building up an effective magnetic field $\left(\boldsymbol{B}_{N}\right)$ acting upon the electrons localized in the QD and hence "stabilizing" their spin. At $\boldsymbol{B}_{\boldsymbol{e x t}}=0$, the orientation of this field could be parallel or antiparallel to the orientation of the averaged electron spin $(\boldsymbol{S})$ (over a large number of excitation events) depending on the sign of both the nuclear gyromagnetic ratio and the electron g-factor. Nuclear fields as large as several Tesla have been reported in experiments on individual $\operatorname{In}(\mathrm{Ga}) \mathrm{As} /$ GaAs QDs. ${ }^{3,37,38}$ Accordingly, we identify $\boldsymbol{B}_{\text {eff }}$ as $\boldsymbol{B}_{\boldsymbol{N}}$.

The lattice nuclei should be most effectively polarized by an electron localized in the QD if there are no other interactions imposed on the electron, e.g., an anisotropic exchange interaction with a hole. Consequently, to explain the experimentally observed fact that even for the case of a neutral exciton, a nonzero nuclear magnetic field builds up in the QD, the exciton formation in a QD is considered as a process of the separate capture of electrons and holes rather than a mechanism according to which the electron and hole are captured into the QD as an entity. The parameter, $\Delta \tau_{\mathrm{e}-\mathrm{h}}$, denotes the difference in the capture times of electrons and holes into the QDs. For excitation with $h v_{e x} \geq E_{H H}$, the electrons and holes undergo transport in the WL prior to capture into the QDs. Electrons are captured first because of their smaller effective mass. ${ }^{16}$ Consequently, $\Delta \tau_{e-h}$ acquires a nonzero value at these excitation conditions, while for the excitation directly into the QDs, $\Delta \tau_{e-h}$ should be negligible.

The efficiency of dynamic nuclear polarization by spinoriented electrons depends on the fraction of time $\left(\Gamma_{e}\right)$ that the QD is occupied with solely an electron. Obviously, $\Gamma_{e}=\Delta \tau_{e-h} / \tau_{r}$, where $\tau_{\mathrm{r}}$ could be regarded as a "recycling" time $\sim P_{e x}{ }^{-1}$, i.e., the average time between two adjacent events of exciton formation in the QD. Accordingly, $\boldsymbol{B}_{N}$ and hence, $\rho_{\mathrm{c}}$, are predicted to be directly proportional to $P_{e x}$ as is indeed observed in our experiments [Fig. 3(a)].

Accordingly, an electron is alone in the QD during $\Delta \tau_{e-h}$ and can then polarize the nuclei. Within a certain number of recycling events the nuclear field, $\boldsymbol{B}_{N}$, builds up. As a result, the electron spin, $S$, is influenced not only by a "destructive" magnetic field, $\omega_{\mathrm{ex}}$, but also by the "stabilizing" nuclear field, $\boldsymbol{B}_{N}$. It is the joint influence of both of these factors that determines the value of the projection of $S$ onto the $z$ axis and hence, $\rho_{\mathrm{c}}$, as measured in the experiment. The present model is very similar to that developed for the exciton pseudospin (see the preceding text) with the only difference being that we consider the electron spin rather than the exciton pseudospin, and the role of $\boldsymbol{B}_{\text {ext }}$ is now played by $\boldsymbol{B}_{\boldsymbol{N}}$.

It is important to note that this model, based on the separate capture of electrons and holes into the QDs, is widely accepted (see, e.g., Refs. 39 and 40 and references therein). Our previous studies ${ }^{16}$ on individual QDs on the same sample, along with denser ensembles of QDs, performed in crossed internal electric and external magnetic fields, have directly demonstrated that the capture processes of electrons and holes into the QD should indeed be separately considered.

Within the framework of our model, the results shown in Figs. 2(b) and 3(a) can be explained. At excitation with $h v_{e x} \geq E_{H H}$, an increase of $P_{e x}$ leads to a more efficient pumping of the nuclear spin (i.e., to a larger $\boldsymbol{B}_{N}$ ) resulting in an increase of $\rho_{\mathrm{c}}$, as previously explained, while no nuclear pumping (and hence, a negligible $\rho_{\mathrm{c}}$ ) is predicted at excitation directly into the QDs, which is in full agreement with the results shown in Fig. 3(a). It should also be pointed out that an alternative model, according to which $\boldsymbol{B}_{\boldsymbol{N}}$ builds-up through the interaction of nuclei with neutral excitons, has 
been proposed. ${ }^{37,41,42}$ Within this approach, the nuclear polarization is determined by the spin-flip assisted radiative recombination of the dark excitons. Although our experimental results emphasize the crucial role played by the separate capture of electrons and holes into the QD, due to the appearance of $\boldsymbol{B}_{\boldsymbol{N}}$, we cannot exclude a possible contribution of dark excitons. Indeed, even at excitation directly into the QD, and at $h v_{e x} \geq E_{H H}$ with the lowest $P_{e x}, \rho_{\mathrm{c}}$ is on the order of several percent [Fig. 3(a)].

It should be mentioned that at excitation with $h v_{e x} \geq E_{H H}$, the nuclei in the WL could, in principle, also be polarized to some degree and their influence on the spin of electrons localized in the QD should be considered. However, this influence is negligible due to the following arguments:

An electron in the WL is free (delocalized) and, hence, its wave function covers a much larger number of nuclei compared to the case of an electron in a QD. Since $\left|\boldsymbol{B}_{\boldsymbol{e}}\right|$ is inversely proportional to the total number of nuclei in the electron's localization volume (see the text above), and taking into account that the typical volume of an individual QD is $\approx 1000$ times smaller than that of the $\mathrm{WL},{ }^{24}$ one could estimate the electron field in the WL to be $\approx 6 \mathrm{mT} /$ $1000=0.006 \mathrm{mT}$, which is essentially less than $B_{L} \approx$ $0.1-0.3 \mathrm{mT}^{22}$ Consequently, nuclear polarization in the WL seems to be unlikely.

(ii) An electron experiences a nuclear field from the polarized nuclei only for the case when these nuclei are located within the electron's localization volume. This is the reason why polarized nuclei in the WL cannot expose an effective magnetic field on an electron localized in the QD.

The decrease of $\rho_{\mathrm{c}}$ recorded at $h v_{e x} \geq E_{H H}$ and $P_{e x}>10$ $\mu \mathrm{W}$ [Fig. 3(a)] should also be discussed. At these excitation powers, the QDs are populated with more than one electronhole pair, as evidenced by the appearance of a broad blueshifted spectral feature (not shown here), which is identified as emission from the QDs excited states. The efficient carrier-carrier scattering at these experimental conditions is believed to explain the reduction of $\rho_{\mathrm{c}}$ recorded at high excitation powers.

To validate our model, i.e., to prove that $\omega_{e x}$ is responsible for the essential electron spin relaxation leading to a small $\rho_{\mathrm{c}}$ at excitation directly into the QDs, we add a second IR laser excitation in addition to the main laser. Since this excitation energy, $h v_{I R}=1.17 \mathrm{eV}$, is well below the QDs transition energies, it does not solely give rise to PL from the QDs. The excitation of just the IR laser gives rise to free holes, due to the excitation of electrons from the valence band into deep levels in the GaAs barriers. ${ }^{15}$ Accordingly, it is predicted that the QDs will become positively charged at dual laser excitation conditions, since the dots become populated with extra holes. These holes will cancel $\omega_{e x}$ and hence, $\rho_{\mathrm{c}}$ is expected to increase, as compared with single laser excitation.

Figure 4(b) shows $\mu$-PL spectra measured with single laser excitation directly into the QDs resolved with respect to their circular polarization with an attained value of $\rho_{\mathrm{c}}=0.07$ [Fig. 4(b)]. Upon the addition of the IR laser with the maximum power available $(\approx 1 \mathrm{~mW})$, a dramatic change in $\rho_{\mathrm{c}}$ is obtained: $\rho_{\mathrm{c}} \approx 0.43$ is derived, i.e., $\approx 6$ times higher, as compared to the case of single laser excitation [Fig. 4(b)]. The evolution of $\rho_{\mathrm{c}}$ as a function of the IR laser excitation power $\left(P_{I R}\right)$ reveals a progressive increase of $\rho_{\mathrm{c}}$ with increasing $P_{I R}$ [inset in Fig. 4(a)], which strongly supports the suggested model. Indeed, an increase of $P_{I R}$ results in a more probable time-averaged population with an extra hole in each QD.

Finally, the typical value of the steady-state concentration of extra holes $\left(n_{h}\right)$, which could be achieved in our experiments with dual laser excitation conditions, should be discussed. It could be calculated as $n_{h}=g_{h} \times \tau_{\mathrm{h}}$, where $\tau_{\mathrm{h}}$ is the capture time of a hole back to deep levels and $g_{h}$ corresponds to the generation rate of the extra holes by the IR laser. The latter is expressed as $g_{h}=\sigma_{\mathrm{h}} \times N_{D L} \times d_{G a A s} \times P_{I R} /$ $h v_{I R}$, where $\sigma_{\mathrm{h}} \approx 10^{-17} \mathrm{~cm}^{2}$ and $N_{D L} \approx 5 \times 10^{13} \mathrm{~cm}^{-3}$ is the optical cross section for the excitation of a free hole in the GaAs barriers and a typical concentration of deep levels in epitaxially grown GaAs material, ${ }^{43}$ respectively, while $d_{\text {GaAs }}=200 \mathrm{~nm}$ stands for the total thickness of the two GaAs barriers. Thus, for $P_{I R}=1 \mathrm{~mW}, g_{h} \approx 5.5 \times 10^{7} \mathrm{~s}^{-1}$ is evaluated. Assuming that the typical value of $\tau_{\mathrm{h}}$ exceeds $\approx 1$ $\mu \mathrm{s},{ }^{44}$ one could derive $n_{h} \geq \approx 55$. This value is on the order of $N \approx 100-140$ previously estimated, thus supporting the idea that, under dual laser excitation, each QD is occupied with an extra hole with a rather large probability $\left(W_{h}\right)$, which is calculated as $W_{h}=n_{h} / N \geq \approx 0.40-0.55$. It is worth mentioning that in the case of $W_{h} \approx 1$, one should expect $\rho_{\mathrm{c}}$

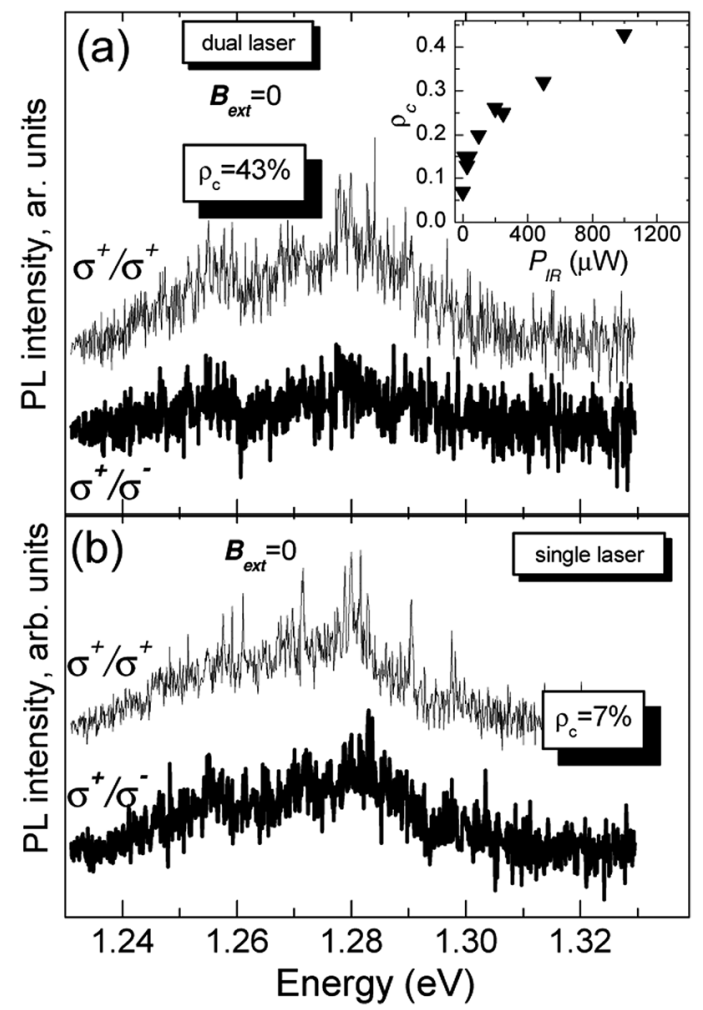

FIG. 4. (a) and (b) show $\mu$-PL spectra of the QDs measured at $\sigma^{+}$cw excitation for dual and single laser excitation conditions, respectively. $h v_{e x}=1.424$ $\mathrm{eV}, P_{e x}=50 \mu \mathrm{W}, h v_{I R}=1.17 \mathrm{eV}, P_{I R}=1 \mathrm{~mW}$, and $B_{\text {ext }}=0 \mathrm{~T}$. The labeling of the thin (thick) lines, $\sigma^{+} / \sigma^{+}\left(\sigma^{+} / \sigma^{-}\right)$, denotes $\sigma^{+}$-polarized excitation and co- (cross-) polarized detection. The spectra have been vertically shifted for clarity. The inset in (a) shows $\rho_{\mathrm{c}}$ as a function of $P_{I R}$ recorded at dual laser excitation with otherwise similar conditions. 
$\approx 100 \%$. Accordingly, the value of $\rho_{\mathrm{c}} \approx 43 \%$ obtained for $P_{I R}=1 \mathrm{~mW}$ in the present experiments [inset of Fig. 4(a)], fits well with the developed model.

\section{CONCLUSIONS}

To conclude, an essential polarization degree, $\rho_{\mathrm{c}} \approx 40 \%$ has been detected in $\mu$-PL spectra of InAs/GaAs QDs ensembles at zero external magnetic field. This phenomenon is explained in terms of the appearance of a nuclear magnetic field in the QDs due to the separate capture of electrons and holes into the dot. An additional IR laser initiates an essential increase of $\rho_{\mathrm{c}}(\approx 6$ times) by changing the charge state of the QDs from neutral to positively charged due to the creation of extra holes in the sample.

\section{ACKNOWLEDGMENTS}

The authors thank P. M. Petroff and W. V. Schoenfeld for the fabrication of the samples. This work was supported by grants from the Swedish Research Council (VR) and the Swedish Foundation for Strategic Research (SSF). The authors are grateful for equipment funding from the Knut and Alice Wallenberg Foundation. E.S.M. acknowledges support from SSF.

${ }^{1}$ Quantum Coherence, Correlation and Decoherence in Semiconductor Nanostructures, edited by T. Takagahara (Elsevier, New York, 2003).

${ }^{2}$ A. V. Khaetskii and Y. V. Nazarov, Phys. Rev. B 61, 12639 (2000).

${ }^{3}$ P.-F. Braun, B. Urbaszek, T. Amand, and X. Marie, Phys. Rev. B 74, 245306 (2006).

${ }^{4}$ E. L. Ivchenko, Pure Appl. Chem. 67, 463 (1995).

${ }^{5}$ P. F. Braun, X. Marie, L. Lombez, B. Urbaszek, T. Amand, P. Renucci, V. K. Kalevich, K. V. Kavokin, O. Krebs, P. Voisin, and Y. Masumoto, Phys. Rev. Lett. 94, 116601 (2005).

${ }^{6}$ Yu. G. Kusrayev, A. V. Koudinov, B. P. Zakharchenya, S. Lee, J. K. Furduna, and M. Dobrowolska, Phys. Rev. B 72, 155301 (2005).

${ }^{7}$ R. I. Dzhioev, H. M. Gibbs, E. L. Ivchenko, G. Khitrova, V. L. Korenev, M. N. Tkachuk, and B. P. Zakharchenya, Phys. Rev. B 56, 13405 (1997).

${ }^{8}$ B. Eble, O. Krebs, A. Lemaître, K. Kowalik, A. Kudelski, P. Voisin, B. Urbaszek, X. Marie, and T. Amand, Phys. Rev. B 74, 081306 (2006).

${ }^{9}$ M. E. Ware, E. A. Stinaff, D. Gammon, M. F. Dotty, A. S. Bracker, D. Gershoni, V. L. Korenev, Ş. C. Bǎdescu, Y. Lyanda-Geller, and T. L. Reinecke, Phys. Rev. Lett. 95, 177403 (2005).

${ }^{10}$ C. W. Lai, P. Maletinsky, A. Badolato, and A. Imamoglu, Phys. Rev. Lett. 96, 167403 (2006).

${ }^{11}$ S. Laurent, M. Senes, O. Krebs, V. K. Kalevich, B. Urbaszek, X. Marie, T. Amand, and P. Voisin, Phys. Rev. B 73, 235302 (2006).

${ }^{12}$ T. Belhadj, C.-M. Simon, T. Amand, P. Renucci, B. Chatel, O. Krebs, A. Lemaître, P. Voisin, X. Marie, and B. Urbaszek, Phys. Rev. Lett. 103, 086601 (2009).

${ }^{13}$ E. S. Moskalenko, L. A. Larsson, and P. O. Holtz, Phys. Rev. B 80, 193413 (2009).

${ }^{14}$ F. Klotz, V. Jovanov, J. Kiering, E. C. Clark, M. Bichler, G. Abstreiter, M. S. Brandt, J. J. Finley, H. Schwager, and G. Giedke, Phys. Rev. B 82, 121307 (2010).

${ }^{15}$ E. S. Moskalenko, V. Donchev, K. F. Karlsson, P. O. Holtz, B. Monemar, W. V. Schoenfeld, J. M. Garcia, and P. M. Petroff, Phys. Rev. B 68, 155317 (2003).

${ }^{16}$ E. S. Moskalenko, L. A. Larsson, M. Larsson, P. O. Holtz, W. V. Schoenfeld, and P. M. Petroff, Nano Lett. 9, 353 (2009).

${ }^{17}$ K. H. Schmidt, G. Medeiros-Ribeiro, J. M. Garcia, and P. M. Petroff, Appl. Phys. Lett. 70, 1727 (1997); J. M. Garcia, T. Mankad, P. O. Holtz, P. J. Wellman, and P.M. Petroff, ibid. 72, 3172 (1998); J. M. Garcia, G. Medeiros-Ribeiro, K. Schmidt, T. Ngo, J. L. Feng, A. Lorke, J. Kotthaus, and P. M. Petroff, ibid. 71, 2014 (1997).

${ }^{18}$ E. S. Moskalenko, K. F. Karlsson, P. O. Holtz, B. Monemar, W. V. Schoenfeld, J. M. Garcia, and P. M. Petroff, Phys. Rev. B 66, 195332 (2002).
${ }^{19}$ E. S. Moskalenko, K. F. Karlsson, V. Donchev, P. O. Holtz, W. V. Schoenfeld, and P. M. Petroff, Appl. Phys. Lett. 84, 4896 (2004).

${ }^{20}$ At excitation with an energy exceeding the band gap of GaAs barriers we can safely disregard the direct absorption into the WL or into the QDs. This is based on the fact that the thickness of each of the two GaAs barriers is 100 $\mathrm{nm}$ while that of the WL is only $0.5 \mathrm{~nm}$. This allows one to evaluate excited volumes of the GaAs (WL) to be $\approx 6.3 \times 10^{-13}\left(1.6 \times 10^{-15}\right) \mathrm{cm}^{3}$.

${ }^{21}$ R. Oulton, A. Greilich, S. Yu. Verbin, R. V. Cherbunin, T. Auer, D. R. Yakovlev, M. Bayer, I. A. Merkulov, V. Stavarache, D. Reuter, and A. D. Wieck, Phys. Rev. Lett. 98, 107401 (2007).

${ }^{22}$ M. I. Dyakonov and V. I. Perel, in Optical Orientation edited by F. Meier and B. P. Zakharchenya (North-Holland, Amsterdam, 1984), Chap. 2.

${ }^{23}$ S. Cortez, O. Krebs, S. Laurent, M. Senes, X. Marie, P. Voisin, R. Ferreira, G. Bastard, J.-M. Gérard, and T. Amand, Phys. Rev. Lett. 89, 207401 (2002).

${ }^{24}$ It is interesting to note that the ratio $\mathrm{IQD}^{\text {above }} / \mathrm{IQD}^{\text {dir }}$ could also be straightforwardly used to evaluate $\mathrm{N} \approx 106$, which is in good agreement with the preceding estimated values of $\mathrm{N}$ ranging from 100 up to 140 . The estimate is obtained on the basis of the idea of simply comparing the absorbing volumes of the WL (VWL) and the QDs (VQD) at excitations above and below WL, respectively. We note that at excitation with $\mathrm{EHH}<$ hvex $<\mathrm{ELH}$ we cannot further disregard the direct absorption into the QDs because their total volume (VQD) is assumed to be equal to $\mathrm{N} \times \mathrm{VSQD}$, where VSQD is the volume of an individual QD evaluated as $\approx 2.2 \times 10^{-18} \mathrm{~cm}^{3}$ relying on its lens-shaped form and base diameter (high) of $\approx 35$ (4.5) nm (Ref. 17), could be comparable to the excited volume of the WL [VWL $\approx 1.6 \times 10^{-15} \mathrm{~cm}^{3}$ (Ref. 20)]. Consequently, $\mathrm{IQD}^{\text {above }}=\mathrm{IQD}^{\mathrm{dir}}+\mathrm{IQ}^{\mathrm{Dc}}$, where IQDc stands for the emission of those excitons which were primarily generated in the excited volume of the WL. As was explained in the text, $\mathrm{IQ}^{\mathrm{Dc}}=\mathrm{G} /(1+\mathrm{R}-1)$, where $\mathrm{R}$ is still considered $\approx 1.4$ and $\mathrm{G}=\mathrm{AVWL}$, where $\mathrm{A}$ accounts for the absorption coefficient in the InAs material and for the laser excitation power. Assuming IQDdir $=A V Q D$, one can easily evaluate $N \approx 106$ from the equation, IQDabove/IQDdir $-1=\mathrm{N}-1 \times(\mathrm{VWL} / \mathrm{VSQD}) \times(1+\mathrm{R}-1)-1$

${ }^{25}$ We can safely neglect mixing of heavy-light hole states because of the strain in self-assembled $\operatorname{In}(\mathrm{Ga}) \mathrm{As} / \mathrm{GaAs}$ QDs (Refs. 3 and 26).

${ }^{26}$ M. Bayer, G. Ortner, O. Stern, A. Kuther, A. A. Gorbunov, A. Forchel, P. Hawrylak, S. Fafard, K. Hinzer, T. L. Reinecke, S. N. Walck, J. P. Reithmaier, F. Klopf, and F. Schäfer, Phys. Rev. B 65, 195315 (2002).

${ }^{27}$ M. Paillard, X. Marie, P. Renucci, T. Amand, A. Jbeli, and J. M. Gérard, Phys. Rev. Lett. 86, 1634 (2001).

${ }^{28}$ To estimate $\rho \mathrm{c}$ we use the expression $\rho_{\mathrm{c}}=\rho_{\mathrm{c}}{ }^{0} /\left(1+\omega_{\mathrm{ex}}{ }^{2} \tau_{\mathrm{d}}{ }^{2}\right)$, derived in Ref. ${ }^{4}$, where $\rho \mathrm{c} 0$ denotes the degree of polarization generated by the laser and is selected to be equal to 1 to estimate the upper bound of $\rho \mathrm{c}$.

${ }^{29}$ A.W. Overhauser, Phys. Rev. 92, 411 (1953).

${ }^{30}$ G. Lampel, Phys. Rev. Lett. 20, 491 (1968).

${ }^{31}$ D. Paget, G. Lampel, B. Sapoval, and V.I. Safarov, Phys. Rev. B 15, 5780 (1977).

${ }^{32}$ V. K. Kalevich, V. L. Korenev, O. M. Fedorova, Pis'ma Z. Eksp. Teor. Fiz. 52, 964 (1990); JETP Lett. 52, 349 (1990).

${ }^{33}$ V. K. Kalevich, and V. L. Korenev, Pis'ma Z. Eksp. Teor. Fiz. 56, 257 (1992); JETP Lett. 56, 253 (1992).

${ }^{34}$ B. P. Zakharchenya, V. K. Kalevich, V. D. Kulkov, V. G. Fleisher, Fiz. Tverd. Tela 23, 1387 (1981); Sov. Phys. Solid State 23, 810 (1981).

${ }^{35}$ R. Iyyapan, Semiconductor Spintronics and Quantum Computation, edited by D. D. Awschalom, N. Samarth, and D. Loss (Springer, Berlin, 2002).

${ }^{36} \mathrm{R}$. Iyyapan, Encyclopedia of Physical Science and Technology, 3rd ed., edited by R. A. Meyers (Academic, San Diego, 2002), Vol. 10, p. 704.

${ }^{37}$ A. I. Tartakovskii, T. Wright, A. Russell, V. I. Fal'ko, A. B. Van'kov, J. Skiba-Szymanska, I. Drouzas, R. S. Kolodka, M. S. Skolnick, P. W. Fry, A. Tahraoui, H.-Y. Liu, and M. Hopkinson, Phys. Rev. Lett. 98, 026806 (2007).

${ }^{38}$ P. Maletinsky, C. W. Lai, A. Badolato, and A. Imamoglu, Phys. Rev. B 75, 035409 (2007).

${ }^{39}$ J. Urayama, T. B. Norris, J. Singh, and P. Bhattacharya, Phys. Rev. Lett. 86, 4930 (2001)

${ }^{40}$ E. C. Le Ru, J. Fack, and R. Murray, Phys. Rev. B 67, 245318 (2003); M. Grundmann and D. Bimberg, Phys. Status Solidi A 164, 297 (1997).

${ }^{41}$ D. Gammon, Al. L. Efros, T. A. Kennedy, M. Rosen, D. S. Katzer, D. Park, S. W. Brown, V. L. Korenev, and I. A. Merkulov, Phys. Rev. Lett. 86, 5176 (2001).

${ }^{42}$ V. L. Korenev, JETP Lett. 70, 129 (1999).

${ }^{43}$ P. Silverberg, P. Omling, and L. Samuelson, Appl. Phys. Lett. 52, 1689 (1988).

${ }^{44}$ D. Paget and P. B. Klein, Phys. Rev. B 34, 971 (1986). 